\title{
An LQ problem for the heat equation on the halfline with Dirichlet boundary control and noise
}

\author{
G. Fabbri*, B. Goldys ${ }^{\dagger}$
}

November 8, 2018

\begin{abstract}
We study a linear quadratic problem for a system governed by the heat equation on a halfline with Dirichlet boundary control and Dirichlet boundary noise. We show that this problem can be reformulated as a stochastic evolution equation in a certain weighted $L^{2}$ space. An appropriate choice of weight allows us to prove a stronger regularity for the boundary terms appearing in the infinite dimensional state equation. The direct solution of the Riccati equation related to the associated non-stochastic problem is used to find the solution of the problem in feedback form and to write the value function of the problem.
\end{abstract}

Key words: heat equation, Dirichlet boundary conditions, boundary noise, boundary control, weighted $L^{2}$ space, analytic semigroup, stochastic convolution, linear quadratic control problem, Riccati equation.

MSC 2000: 35G15, 37L55, 49N10.

\section{Introduction}

In this paper we are concerned with a linear quadratic control problem for a heat equation on the halfline $[0, \infty)$ with Dirichlet boundary control and boundary noise. More precisely, for fixed $0 \leq \tau<T$, we deal with the equation

$$
\begin{cases}\frac{\partial}{\partial t} y(t, \xi)=\frac{\partial^{2}}{\partial \xi^{2}} y(t, \xi) & t \in[\tau, T], \xi>0, \\ y(t, 0)=u(t)+\dot{W}(t) & t \in[\tau, T] \\ y(\tau, \xi)=x_{0}(\xi) & \xi>0 .\end{cases}
$$

where $W$ is a one dimensional Brownian motion and $u$ is a square-integrable control. Let us recall that a deterministic boundary control problem

$$
\begin{cases}\frac{\partial}{\partial t} z(t, \xi)=\frac{\partial^{2}}{\partial \xi^{2}} z(t, \xi) & t \in[\tau, T], \xi>0 \\ z(t, 0)=u(t) & t \in[\tau, T], \\ z(\tau, \xi)=x_{0}(\xi) & \xi>0\end{cases}
$$

\footnotetext{
${ }^{*}$ School of Mathematics and Statistics, UNSW, Sydney, e-mail: fabbri@maths.unsw.edu.au, supported by the ARC Discovery project DP0558539.

${ }^{\dagger}$ School of Mathematics and Statistics, UNSW, Sydney, e-mail: goldys@maths.unsw.edu.au, supported by the ARC Discovery project DP0558539.
} 
is well understood, see for example [3], [13]). Denoting by $A_{0}$ the Dirichlet Laplacian in $L^{2}(0, \infty)$ and by $D$ the Dirichlet map (defined as $D_{\lambda_{0}}$ in (10) below), we can rewrite (2) in the form

$$
z(t)=e^{t A_{0}} x_{0}+\left(\lambda_{0}-A_{0}\right) \int_{0}^{t} e^{(t-s) A_{0}} D u(s) d s,
$$

and it is easy to show that $z(t) \in L^{2}(0, \infty)$ for all $t \geq 0$. Therefore, the process

$$
X(t)=e^{t A_{0}} x_{0}+\left(\lambda_{0}-A_{0}\right) \int_{0}^{t} e^{(t-s) A_{0}} D u(s) d s+\left(\lambda_{0}-A_{0}\right) \int_{0}^{t} e^{(t-s) A_{0}} D d W(s)
$$

seems to be a good candidate for a solution to (11). However, it was shown in [7] that the process $X$ is not $L^{2}$-valued. More precisely, it was shown that the solution to (1) considered on a finite interval and for $u=0$, when rewritten in the form (3), is well defined in a negative Sobolev space $H^{-\alpha}$ for $\alpha>\frac{1}{2}$ only. It is easy to see that the same conclusion holds in the case of halfline. Then it was shown in [2, see also [4], that the process $X$ can be defined pointwise on $(0, \infty)$ and it takes values in a weighted space $L^{2}\left(0, \infty ; \xi^{1+\theta} d \xi\right)$. This fact was used to study some properties of the process $X$ (in fact in the aforementioned papers more general nonlinear equations are studied) but the problem is not reformulated as a stochastic evolution equation in $L^{2}\left(0, \infty ; \xi^{1+\theta} d \xi\right)$ and therefore advantages of using the weighted space are somewhat limited.

Following the idea of Krylov [12] we introduce the weighted spaces $\mathcal{H}_{\rho}=L^{2}((0, \infty) ; \rho(\xi) d \xi)$, where for $\theta \in(0,1)$ we have

$$
\rho(\xi)=\xi^{1+\theta} \quad \text { or } \quad \rho(\xi)=\min \left(1, \xi^{1+\theta}\right), \quad \xi \geq 0 .
$$

It was proved in [11] and [12] that the Dirichlet Laplacian $A_{0}$ defined on $L^{2}(0, \infty)$ extends to a generator $A$ of an analytic semigroup $\left(e^{t A}\right)$ on $\mathcal{H}_{\rho}$. We will show that the Dirichlet map takes values in $\operatorname{dom}\left((-A)^{\alpha}\right)$ for a certain $\alpha>\frac{1}{2}$ and therefore equation (3), when considered in $\mathcal{H}_{\rho}$, can be given a form

$$
X(t)=e^{t A} x_{0}+\int_{0}^{t} e^{(t-s) A}\left(\lambda_{0}-A\right) D u(s) d s+\int_{0}^{t} e^{(t-s) A}\left(\lambda_{0}-A_{0}\right) D d W(s)
$$

that is, we will study a controlled evolution equation

$$
\left\{\begin{array}{l}
\mathrm{d} x(t)=(A x(t)+B u(t)) \mathrm{d} t+B \mathrm{~d} W(t) \\
x(\tau)=x_{0} \in \mathcal{H}_{\rho}
\end{array}\right.
$$

for $B=\left(\lambda_{0}-A\right) D$. This fact is a starting point for our analysis of the linear quadratic control problem (1). We will demonstrate that the control problem (4) when considered in the space $\mathcal{H}_{\rho}$ can be solved using classical by now techniques presented, for example, in [3]. Let us emphasize that while focus of this paper is on the most interesting case of boundary control and boundary noise a more general control problem

$$
\left\{\begin{array}{l}
\mathrm{d} x(t)=\left(A x(t)+B u_{1}(t)+v(t)\right) \mathrm{d} t+B \mathrm{~d} W(t)+d W_{1}(t) \\
x(\tau)=x_{0} \in \mathcal{H}_{\rho}
\end{array}\right.
$$

with spatially distributed noise $W_{1}$ and control $v$ might be easily considered using the same technique. 
Let us note that if the boundary conditions are of Neumann type then the analogue of equation (11) has a solution in $L^{2}(0, \infty)$ and has been studied intensely (also for more general parabolic equations with boundary noise), see for example [7], 17], [8], [9].

We study the linear quadratic problem characterized by the cost functional

$$
J\left(\tau, x_{0}, u\right)=\mathbb{E}\left[\int_{\tau}^{T}|C x(t)|_{Y}^{2}+|u(t)|_{\mathbb{R}}^{2} \mathrm{~d} t+\langle G x(T), x(T)\rangle_{\mathcal{H}_{\rho}}\right]
$$

and governed by a state equation of the form (4). the operator $C$ that appears in (6) is in $\mathcal{L}\left(\mathcal{H}_{\rho} ; Y\right)$ for a certain Hilbert space $Y$ and $G \in \mathcal{L}\left(\mathcal{H}_{\rho} ; \mathcal{H}_{\rho}\right)$ is symmetric and positive. The direct solution of the Riccati equation related to a linear quadratic problem driven by a stochastic equation different from ours was studied in the Neumann case (non-weighted setting) in [10] (see also [1] and [5] for the control inside the domain case $(\alpha=1)$ ). Our approach is different from the one used in the aforementioned works since we directly use the solution of the Riccati equation for the "associated" deterministic problem.

The deterministic linear quadratic problem associated to ours is that characterized by the state equation

and the functional

$$
\dot{x}(t)=A x(t)+B u(t)
$$

$$
\int_{\tau}^{T}\left(|C x|_{Y}^{2}+|u|_{\mathbb{R}}^{2}\right) \mathrm{d} t+\langle G x(T), x(T)\rangle_{\mathcal{H}_{\rho}}
$$

It is well known, see [3] and Section 3 below, that the solution to the linear quadratic problem given above is determined by the operator-valued function $P:[0, T] \rightarrow \mathcal{L}\left(\mathcal{H}_{\rho}, \mathcal{H}_{\rho}\right)$ which solves the so-called Riccati equation

$$
\left\{\begin{array}{l}
P^{\prime}(t)=-A^{*} P(t)-P(t) A^{*}-C^{*} C+P(t) A B B^{*} A^{*} P(t) \\
P(T)=G
\end{array}\right.
$$

Such a problem has been intensely studied (see [3] and [14] and the references therein). We will refer in particular to the direct solution approach and we will use the formalism introduced in Section 2.2. of [3]. We show that the Riccati equation (8) has a unique solution $P$ in the space $C_{s, \alpha}([0, T] ; \Sigma(\mathcal{H})$ ) (see Definition [3.2). Let us note that in the deterministic case the minimum of the cost functional (7) is given by $\left\langle P(\tau) x_{0}, x_{0}\right\rangle_{\mathcal{H}}$.

In the study of the problem with boundary noise some of the tools and the results of the deterministic case, as the properties of the elements of $C_{s, \alpha}\left([0, T] ; \Sigma\left(\mathcal{H}_{\rho}\right)\right)$ and the solution of (8), are still useful. It is possible to express the value function and the optimal feedback in terms of $P$. A term due to the noise appears in the expression of the minimal cost and we have that (Theorem 3.7):

$$
\begin{aligned}
V\left(\tau, x_{0}\right)=\inf _{u \in \mathcal{U}_{\tau}} J & \left(\tau, x_{0}, u\right)= \\
& =\left\langle P(\tau) x_{0}, x_{0}\right\rangle+\int_{\tau}^{T} \frac{1}{2}\left\langle\left(\left(\lambda_{0}-A\right) D(1)\right), P(s)\left(\left(\lambda_{0}-A\right) D(1)\right)\right\rangle_{\mathcal{H}} \mathrm{d} s .
\end{aligned}
$$

\section{The heat equation in $\mathcal{H}_{\rho}$}

\subsection{Notation}

We will work in a weighted space $\mathcal{H}_{\rho}=L^{2}([0, \infty) ; \rho(\xi) d \xi)$, where either $\rho(\xi)=\xi^{1+\theta} \wedge 1$ or $\rho(\xi)=\xi^{1+\theta}$ for some $\theta \in(0,1)$ and $\xi \geq 0$. All the results proved in the sequel are valid for 
both weights and therefore, in order to simplify notations we will use the same notation $\mathcal{H}=\mathcal{H}_{\rho}$ for both weights. Let us recall that $f \in \mathcal{H}$ if and only if

$$
\int_{0}^{\infty} f^{2}(\xi) \rho(\xi) \mathrm{d} \xi<\infty
$$

and $\mathcal{H}$ is a Hilbert space with the scalar product

$$
\langle\phi, \psi\rangle_{\mathcal{H}}=\int_{0}^{\infty} \phi(\xi) \psi(\xi) \rho(\xi) \mathrm{d} \xi \quad \text { for all } \phi, \psi \in \mathcal{H} .
$$

Given $\lambda>0$, the Dirichlet map $D_{\lambda}$ is defined as follows:

$$
D_{\lambda}(a)=\phi \Longleftrightarrow\left\{\begin{array}{l}
\left(\lambda-\partial_{x}^{2}\right) \phi(\xi)=0 \quad \text { for all } \xi>0 \\
\phi(0)=a
\end{array}\right.
$$

so $D_{\lambda}(a)=a \psi_{\lambda}$ where

$$
\left\{\begin{array}{l}
\psi_{\lambda}: \mathbb{R}^{+} \rightarrow \mathbb{R} \\
\psi_{\lambda}: \xi \mapsto e^{-\sqrt{\lambda} \xi}
\end{array}\right.
$$

Clearly $\psi_{\lambda} \in \mathcal{H}$.

It is well known that for every $x_{0} \in L^{2}(0, \infty)$ the solution $y$ to the heat equation with zero Dirichlet boundary condition

$$
\begin{cases}\frac{\partial}{\partial t} y(t, \xi)=\frac{\partial^{2}}{\partial \xi^{2}} y(t, \xi) & t>0, \xi>0 \\ y(t, 0)=0 & t \geq 0 \\ y(0, \xi)=x_{0}(\xi) & \xi>0 .\end{cases}
$$

is given by the following well known expression

$$
y(t, \xi)=\int_{0}^{\infty} k(t, \xi, \eta) x_{0}(\eta) \mathrm{d} \eta
$$

where

$$
k(t, \xi, \eta)=\frac{1}{\sqrt{4 \pi t}}\left(e^{-\frac{(\xi-\eta)^{2}}{4 t}}-e^{-\frac{(\xi+\eta)^{2}}{4 t}}\right), \eta, \xi \geq 0 .
$$

This formula defines the corresponding heat semigroup $T(t) x_{0}=y(t)$ in $L^{2}(0, \infty)$. It is also well known that $(T(t))$ is a symmetric $C_{0}$-semigroup of contractions on $L^{2}(0, \infty)$.

\subsection{Properties of the heat semigroup on $\mathcal{H}$}

Proposition 2.1. For each of the weights $\rho(\xi)$ considered above, the heat semigroup $(T(t))$ extends to a bounded $C_{0}$ semigroup $\left(e^{t A}\right)_{t \geq 0}$ on $\mathcal{H}$ with generator $A: \operatorname{dom}(A) \rightarrow \mathcal{H}$. The semigroup $\left(e^{t A}\right)_{t \geq 0}$ is analytic.

Proof. The case $\rho(\xi)=\xi^{1+\theta}: \mathcal{H}=L^{2}\left([0, \infty), \xi^{1+\theta} d \xi\right)$.

Let $f \in L^{2}(0, \infty)$. Then by Theorem 2.5 in [12] there exists $C>0$ independent of $f$ and such that

$$
\left|e^{t A} f\right|_{\mathcal{H}} \leq C|f|_{\mathcal{H}}, \quad t \geq 0
$$


Since $L^{2}(0, \infty)$ is dense in $\mathcal{H}, e^{t A}$ can be extended to $\mathcal{H}$ and the strong continuity follows by standard arguments. Let $A_{0}$ be the generator of $(T(t))$ in $L^{2}(0, \infty)$ and let $\mathcal{D}=\operatorname{dom}\left(A_{0}\right) \cap \mathcal{H} \subset$ $\mathcal{H}$. Clearly

$$
e^{t A} \mathcal{D} \subset \mathcal{D}, \quad t \geq 0
$$

and $\mathcal{D}$ is dense in $\mathcal{H}$. Therefore $\mathcal{D}$ is a core for the generator $A$ of $\left(e^{t A}\right)$ in $\mathcal{H}$. If $f \in \mathcal{D}$ then

$$
A e^{t A} f=\frac{\partial^{2}}{\partial \xi^{2}} T(t) f
$$

and again by Theorem 2.5 in [12] we have

$$
\left|\frac{\partial^{2}}{\partial \xi^{2}} T(t) f\right|_{\mathcal{H}} \leq \frac{C}{t}|f|_{\mathcal{H}} .
$$

Since $\mathcal{D}$ is a core for the generator $A$ in $\mathcal{H}$, the above estimate can be extended to any $f \in \mathcal{H}$ and therefore

$$
\left|A e^{t A} f\right|_{\mathcal{H}} \leq \frac{C}{t}|f|_{\mathcal{H}} \quad f \in \mathcal{H}
$$

The last inequality is equivalent to the analyticity of the semigroup $\left(e^{t a}\right)$ in $\mathcal{H}$. follows. The case $\rho(\xi)=1 \wedge \xi^{1+\theta}: \mathcal{H}=L^{2}\left([0, \infty), 1 \wedge \xi^{1+\theta} d \xi\right)$.

Let $x \in C_{0}^{\infty}(0, \infty)$ and $t \leq T$. Then the functions $x_{1}=x I_{[0,1]}$ and $x_{2}$ are in $L^{2}(0, \infty)$ and $\mathcal{H}_{\rho}$ for both weights $\rho$. It follows that

$$
\begin{aligned}
\mid\left(\left.T(t) x\right|_{\mathcal{H}}\right. & \leq\left|T(t)\left(\chi_{[0,1]} x\right)\right|_{\mathcal{H}}+\left|T(t)\left(\chi_{(1,+\infty)} x\right)\right|_{\mathcal{H}} \\
& \leq\left|T(t)\left(\chi_{[0,1]} x\right)\right|_{L_{\xi^{1+\theta}}^{2}}+\left|T(t)\left(\chi_{(1,+\infty)} x\right)\right|_{L^{2}(0,+\infty)} \\
& \leq C|x|_{\mathcal{H}}
\end{aligned}
$$

for a certain $C>0$. The fact that $C$ does not depend on $t \leq T$ is a consequence of the $C_{0}$ property of $T_{t}$ on $L_{\xi^{1+\theta}}^{2}$ (showed in the first part of the proof) and on $L^{2}(0, \infty)$. Therefore $(T(t))$ has an extension to a semigroup $\left(e^{t A}\right)$ on $\mathcal{H}$ and the $C_{0}$-property follows by standard arguments. Similar arguments yield analyticity of $\left(e^{t A}\right)$.

Lemma 2.2. Assume that $\lambda>0$ and $r>0$. Then

$$
\psi_{\lambda} \in \operatorname{dom}\left((r-A)^{\alpha}\right) \quad \text { for all } \quad \alpha \in\left[0, \frac{1}{2}+\frac{\theta}{4}\right) .
$$

In particular $D_{\lambda} \in \mathcal{L}\left(\mathbb{R} ; \operatorname{dom}\left((\lambda-A)^{\alpha}\right)\right)$ for all $\alpha \in\left[0, \frac{1}{2}+\frac{\theta}{4}\right)$.

Proof. We consider the case of $\rho(\xi)=\xi^{1+\theta}$ only. The other case may be proved by similar if somewhat simpler arguments.

Note first that if $\psi_{\lambda} \in(\operatorname{dom}(A), \mathcal{H})_{2, \sigma}$ then $\psi_{\lambda} \in \operatorname{dom}\left((r-A)^{\alpha}\right)$ for all $\alpha \in(0,1-\sigma) 1$, see for example Theorem 11.5.1 in [16]. Hence the claim will follow if we show that $\psi_{\lambda} \in$ $(\operatorname{dom}(A), \mathcal{H})_{2, \sigma}$ for

$$
\frac{1}{2}-\frac{\theta}{4}<\sigma<\frac{1}{2}
$$

\footnotetext{
${ }^{1}(\operatorname{dom}(A), \mathcal{H})_{2, \sigma}$ denotes the real interpolation space
} 
By Theorem 10.1 of [15]) $\psi_{\lambda} \in(\operatorname{dom}(A), \mathcal{H})_{2, \sigma}$ if and only if

$$
\int_{0}^{\infty} t^{2 \sigma-3}\left|\left(e^{t A}-I\right) \psi_{\lambda}\right|_{\mathcal{H}}^{2} d t<\infty
$$

and taking into account (15) it is enough to show that

$$
I:=\int_{0}^{1} t^{2 \sigma-3}\left|\left(e^{t A}-I\right) \psi_{\lambda}\right|_{\mathcal{H}}^{2}<\infty
$$

To show (17) we will use (12) and (13) and the definition of $\psi_{\lambda}$. Denoting by $\mathbf{N}$ the cumulative distribution function of the standard normal distribution, we obtain

$$
\begin{aligned}
I & =\int_{0}^{1} t^{2 \sigma-3} \int_{0}^{\infty} \xi^{1+\theta}\left|\left(e^{t A}-I\right) \psi_{\lambda}(\xi)\right|^{2} d \xi d t \\
& =\int_{0}^{1} t^{2 \sigma-3} \int_{0}^{\infty} \xi^{1+\theta}\left(\int_{0}^{\infty} \frac{e^{-\frac{(\xi-\eta)^{2}}{4 t}}}{\sqrt{4 p t}} e^{-\lambda \eta} d \eta-\int_{0}^{\infty} \frac{e^{-\frac{(\xi+\eta)^{2}}{4 t}}}{\sqrt{4 p t}} e^{-\lambda \eta} d \eta-e^{\lambda \xi}\right)^{2} d \xi d t \\
& =\int_{0}^{1} t^{2 \sigma-3} \int_{0}^{\infty} \xi^{1+\theta}\left(e^{-\lambda \xi} e^{\lambda^{2} t} \mathbf{N}\left(\frac{\xi}{\sqrt{2 t}}-\lambda \sqrt{t}\right)-e^{\lambda \xi} e^{\lambda^{2} t}\left(1-\mathbf{N}\left(\frac{\xi}{\sqrt{2 t}}+\lambda \sqrt{t}\right)\right)-e^{\lambda \xi}\right)^{2} d \xi d t \\
& \leq 2\left(I_{1}+I_{2}+I_{3}\right)
\end{aligned}
$$

where $I_{1}, I_{2}$ and $I_{3}$ are respectively

$$
\begin{aligned}
I_{1} & :=\int_{0}^{1} t^{2 \sigma-3} \int_{0}^{+\infty} \xi^{1+\theta}\left[e^{-\lambda \xi}\left(e^{\lambda^{2} t}-1\right) \mathbf{N}\left(\frac{\xi}{\sqrt{2 t}}-\lambda \sqrt{2 t}\right)\right]^{2} \mathrm{~d} \xi \mathrm{d} t \\
I_{2} & :=\int_{0}^{1} t^{2 \sigma-3} \int_{0}^{+\infty} \xi^{1+\theta}\left[e^{-\lambda \xi}\left(\mathbf{N}\left(\frac{\xi}{\sqrt{2 t}}-\lambda \sqrt{2 t}\right)-1\right)\right]^{2} \mathrm{~d} \xi \mathrm{d} t \\
I_{3} & :=\int_{0}^{1} t^{2 \sigma-3} \int_{0}^{+\infty} \xi^{1+\theta}\left[e^{\lambda \xi} e^{\lambda^{2} t}\left(1-\mathbf{N}\left(\frac{\xi}{\sqrt{2 t}}+\lambda \sqrt{2 t}\right)\right)\right]^{2} \mathrm{~d} \xi \mathrm{d} t
\end{aligned}
$$

Since for $t \in[0,1]$ we have $\left|e^{\lambda^{2} t}-1\right| \leq\left(e^{\lambda^{2}}-1\right) t$ we find that $I_{1}$ converges for every $\sigma>0$. $I_{3}$ can be estimated, using that the standard estimate

$$
(1-\mathbf{N}(s)) \leq \frac{1}{s} \frac{e^{-s^{2} / 2}}{\sqrt{2 \pi}}
$$

as follows:

$$
\begin{aligned}
I_{3} & \leq \int_{0}^{1} t^{2 \sigma-3} \int_{0}^{+\infty} \xi^{1+\theta} e^{2 \lambda \xi} e^{2 \lambda^{2} t} \frac{2 t}{\xi^{2}} e^{-\xi^{2} /(2 t)} \mathrm{d} \xi \mathrm{d} t \\
& \leq C_{1} \int_{0}^{1} t^{2 \sigma-2} \int_{0}^{+\infty} \xi^{-1+\theta} e^{2 \lambda \xi-\xi^{2} /(2 t)} \mathrm{d} \xi \mathrm{d} t \\
& =C_{1} \int_{0}^{1} t^{2 \sigma-2} \int_{0}^{+\infty} y^{-1+\theta} t^{\frac{\theta-1}{2}} e^{2 \lambda y \sqrt{t}-y^{2} / 2} t^{1 / 2} \mathrm{~d} \xi \mathrm{d} t \\
& \leq C_{1}\left(\int_{0}^{1} t^{2 \sigma-2+\frac{\theta}{2}} \mathrm{~d} t\right)\left(\int_{0}^{+\infty} y^{-1+\theta} e^{2 \lambda y-y^{2} / 2} \mathrm{~d} \xi\right)<\infty
\end{aligned}
$$

where the finiteness of the first term follows from (15). The estimate for $I_{2}$ can be obtained in a similar way. 


\subsection{Properties of the solution of the state equation}

Let $W$ be a real Brownian motion on a probability space $(\Omega, \mathcal{F}, \mathbb{P})$ and let $\left(\mathcal{F}_{t}\right)$ denote the natural filtration of $W$. We need to give a rigorous meaning to equation (1). To this end we will assume in the sequel that

$$
\lambda_{0}>0 \quad \text { and } \quad \alpha \in\left(\frac{1}{2}, \frac{1}{2}+\frac{\theta}{4}\right)
$$

are fixed. We will denote by $D$ the operator $D_{\lambda_{0}} \in \mathcal{L}\left(\mathbb{R} ; D\left(\left(\lambda_{0}-A\right)^{\alpha}\right)\right)$ and $\psi_{\lambda_{0}}=D_{\lambda_{0}}(1)$. By Proposition 2.1 the semigroup $\left(e^{t A}\right)$ is analytic and therefore for any $\gamma \geq 0$

$$
\left\|\left(\lambda_{0}-A\right)^{\gamma} e^{t A}\right\|_{\mathcal{H}} \leq M_{\gamma} t^{-\gamma} \quad \text { for all } t \in(0, T],
$$

see for example [18] (Theorem 6.13 page 75). By Lemma 2.2 the operator $B=\left(\lambda_{0}-A\right) D$ : $\mathbb{R} \rightarrow \mathcal{H}^{\alpha-1}$ is bounded 2 . Moreover, for $t>0$ the operator

$$
A e^{t A} D_{\lambda_{0}}=\left(\lambda_{0}-A\right)^{1-\alpha} e^{t A}\left(\lambda_{0}-A\right)^{\alpha} D_{\lambda_{0}}: \mathbb{R} \rightarrow \mathcal{H}
$$

is bounded as well. We will write $e^{t A} B=A e^{t A} D_{\lambda_{0}}$ Now, we reformulate equation equation (1), still formally, as a stochastic evolution equation in $\mathcal{H}$ :

$$
\left\{\begin{array}{l}
\mathrm{d} x(t)=(A x(t)+B u(t)) \mathrm{d} t+B \mathrm{~d} W(t) \\
x(\tau)=x_{0} \in \mathcal{H}
\end{array}\right.
$$

where the control $u$ is chosen in the set $M_{W}^{2}(\tau, T ; \mathbb{R})$ of progressively measurable processes endowed with the norm

$$
\|u\|_{M_{W}^{2}}^{2}=\mathbb{E} \int_{\tau}^{T}|u(t)|^{2} \mathrm{~d} t<\infty .
$$

The next two results show that we can give a meaning to (19).

Theorem 2.3. For all $\gamma<2 \alpha-1$ the following holds.

(i) The operator $t \rightarrow e^{t A} B: \mathbb{R} \rightarrow \mathcal{H}$ is bounded for each $t>0$ and the function

$$
t \rightarrow e^{t A} B a
$$

is continuous for every $a \in \mathbb{R}$.

(ii)

$$
\int_{0}^{T} s^{-\gamma}\left\|\left(\lambda_{0}-A\right) e^{s A} \psi_{\lambda_{0}}\right\|_{\mathcal{H}}^{2} \mathrm{~d} s<\infty
$$

(iii) For every $T>\tau \geq 0$ the process

$$
W_{A}(t)=\int_{\tau}^{t} e^{(t-s) A} B \mathrm{~d} W(s), \quad t \in[\tau, T]
$$

is well defined, belongs to $C\left([\tau, T] ; L^{2}(\Omega ; \mathcal{H})\right)$ and has continuous trajectories in $\mathcal{H}$.

\footnotetext{
${ }^{2}$ For $\beta>0$ the space $\mathcal{H}^{-\beta}$ is defined as a completion of $\mathcal{H}$ with respect to the norm $|x|_{-\beta}=\left|\left(\lambda_{0}-A\right)^{-\beta} x\right|$
} 
Proof. (i) It follows immediately from the definition of $B$ and Lemma 2.2 since

$$
e^{t A} B a=a\left(\lambda_{0}-A\right)^{1-\alpha} e^{t A}\left(\lambda_{0}-A\right)^{\alpha} \psi_{\lambda_{0}}, \quad a \in \mathbb{R}
$$

(ii) (By 21) and (18) we have for $\alpha \in\left(\frac{1}{2}, \frac{1}{2}+\frac{\theta}{4}\right)$

$$
\left\|\left(\lambda_{0}-A\right) e^{s A} \psi_{\lambda_{0}}\right\|_{H S}^{2}=\left|\left(\lambda_{0}-A\right)^{1-\alpha} e^{s A}\left(\lambda_{0}-A\right)^{\alpha} \psi_{\lambda_{0}}\right|^{2} \leq \frac{C}{s^{2(1-\alpha)}}\left|\left(\lambda_{0}-A\right)^{\alpha} \psi_{\lambda_{0}}\right|^{2}
$$

and the estimate (20) follows immediately for a certain $\gamma<2 \alpha-1$.

(iii) Using (20) with $\gamma=0$ we find immediately that, for every $t \geq 0, W_{A}(t)$ is well defined and (see for example [6] Proposition 4.5 page 91)

$$
\mathbb{E}\left|W_{A}(t)\right|_{\mathcal{H}}^{2}=\int_{\tau}^{t}\left|e^{s A}((\lambda-A) D)\right|_{H S}^{2} \mathrm{~d} s<\infty .
$$

Such an estimate gives also, through standard arguments, the mean square continuity. The continuity follows from (20) for $\gamma>0$ using a factorization argument as in [7] Theorem 2.3 page 174 .

Lemma 2.4. Let $T>0$ be fixed, $\lambda>0$ and $u \in M_{W}^{2}(\tau, T ; \mathbb{R})$. Then the process

$$
I(t)=\int_{\tau}^{t} e^{(t-s) A} B u(s) \mathrm{d} s, \quad t \leq T,
$$

is well defined, $I \in M_{W}^{2}(\tau, T ; \mathcal{H})$, and there exists $C>0$ such that

$$
\mathbb{E}\|I\|_{M_{W}^{2}}^{2} \leq C\|u\|_{M_{W}^{2}}^{2} .
$$

Moreover, $I$ is in $C\left(\tau, T ; L^{2}(\Omega, \mathcal{H})\right)$ and has continuous trajectories.

Proof. The first part of the Lemma follow from (21) by standard arguments. The mean-square continuity and continuity of $I$ follows from (18) and Hölder inequality (since $\alpha>1 / 2$ ) in the expression

$$
I(t)=\int_{\tau}^{t}\left[\left(\lambda_{0}-A\right)^{1-\alpha} e^{(t-s) A}\right]\left[\left(\lambda_{0}-A\right)^{\alpha} D u(s)\right] \mathrm{d} s .
$$

and the claim follows.

Definition 2.5. Let $u \in M_{W}^{2}$. An $\mathcal{H}$-valued predictable process $x$, defined on $[0, T]$ is called a mild solution of (19) if

$$
\mathbb{P}\left[\int_{\tau}^{T}|x(s)|^{2} \mathrm{~d} s<\infty\right]=1
$$

and

$$
x(t)=e^{(t-\tau) A} x_{0}+\int_{\tau}^{t} e^{(t-s) A} B u(s) \mathrm{d} s+\int_{\tau}^{t} e^{(t-s) A} B \mathrm{~d} W(s)
$$

Theorem 2.6. Equation (19) has a unique mild solution $x \in C\left(\tau, T ; L^{2}(\Omega, \mathcal{H})\right)$. Moreover, $x$ has continuous trajectories $\mathbb{P}$-a.s. If $u=0$ then equation (19) defines a Markov process in $\mathcal{H}$.

Proof. The properties of the stochastic convolution term come from Lemma 2.3, those of $\int_{\tau}^{t} e^{(t-s) A} B u(s)$ from Lemma 2.4. The Markov property can be proved with standard arguments (see for example [6] Theorem 9.8 page 249). 


\subsection{The approximating equation}

Let $\mathcal{I}_{n} \stackrel{\text { def }}{=}\left(n(n-A)^{-1}\right)^{2}$. We will approximate $x$ using

$$
x_{n} \stackrel{\text { def }}{=} \mathcal{I}_{n} x .
$$

We have that

$$
x_{n} \stackrel{C\left([\tau, T] ; L^{2}(\Omega, \mathcal{H})\right)}{\longrightarrow} x .
$$

We use it to obtain more regularity and to guarantee the existence of a strong solution and then to be able to apply the Ito's rule (Proposition 3.6). From Proposition 2.6 we know that $x_{n} \in C\left([\tau, T] ; L^{2}\left(\Omega, \operatorname{dom}\left(A^{2}\right)\right)\right)$. We have $B_{n}:=\mathcal{I}_{n} B \in \mathcal{L}(\mathbb{R} ; \operatorname{dom}(A))$ and then $B_{n} u \in$ $M_{W}^{2}(\tau, T ; \operatorname{dom}(A))$. Furthermore, $x_{n}$ satisfies the following stochastic differential equation:

$$
\left\{\begin{array}{l}
\mathrm{d} x_{n}(t)=\left(A x_{n}(t)+B_{n} u(t)\right) \mathrm{d} t+B_{n} \mathrm{~d} W(t) \\
x_{n}(\tau)=\mathcal{I}_{n} x_{0}
\end{array}\right.
$$

in strong (an then mild) sense (see [6] Section 6.1). So we have

$$
x_{n}(t)=\mathcal{I}_{n} x_{0}+\int_{\tau}^{t} A x_{n}(s) \mathrm{d} s+\int_{\tau}^{t} B_{n} u(s) \mathrm{d} s+\int_{\tau}^{t} B_{n} \mathrm{~d} W(s)
$$

\section{The linear quadratic problem}

Let us recall that we work under the assumption

$$
\frac{1}{2}<\alpha<\frac{1}{2}+\frac{\theta}{4}
$$

We consider another Hilbert space $Y$, an operator $C \in \mathcal{L}(\mathcal{H} ; Y)$ and a symmetric and positive $G \in \mathcal{L}(\mathcal{H} ; \mathcal{H})$. For a fixed $T>0$ we define the set of the admissible controls as $\mathcal{U}_{\tau}=$ $M_{W}^{2}(\tau, T ; \mathbb{R})$. We consider the linear quadratic optimal control problem governed by equation (19) and quadratic cost functional (to be minimized)

$$
J\left(\tau, x_{0}, u\right):=\mathbb{E}\left[\int_{\tau}^{T}\left(|C x(t)|_{Y}^{2}+|u(t)|_{\mathbb{R}}^{2}\right) \mathrm{d} t+\langle G x(T), x(T)\rangle\right] .
$$

The value function of the problem is

$$
V\left(\tau, x_{0}\right):=\inf _{u \in \mathcal{U}_{\tau}} J\left(\tau, x_{0}, u\right)
$$

We consider now the "associated" deterministic linear quadratic problem. It is characterized by the state equation

$$
\left\{\begin{array}{l}
\dot{x}(t)=A x(t)+B u(t) \\
x(\tau)=x_{0}
\end{array}\right.
$$

by the set of admissible controls $\mathcal{U}_{D E T}:=L^{2}(\tau, T ; \mathbb{R})$ and by the functional

$$
J_{D E T}\left(\tau, x_{0}, u\right):=\int_{\tau}^{T}\left(|C x(t)|_{Y}^{2}+|u(t)|_{\mathbb{R}}^{2}\right) \mathrm{d} t+\langle G x(T), x(T)\rangle .
$$

In what follows we we will use the following notations. 
Notation 3.1. $\quad \Sigma(\mathcal{H})=\{T \in \mathcal{L}(\mathcal{H} ; \mathcal{H}):$ Thermitian $\}$

$$
\begin{aligned}
& \Sigma^{+}(\mathcal{H})=\{T \in \Sigma(\mathcal{H}):\langle T x, x\rangle \geq 0 \text { for all } x \in \mathcal{H}\} \\
& C_{s}([0, T] ; \Sigma(\mathcal{H}))=\{F:[0, T] \rightarrow \Sigma(\mathcal{H}): \text { F strongly continuous }\}
\end{aligned}
$$

Note that ([3] page 137) for every $P \in C_{s}([0, T] ; \Sigma(\mathcal{H}))$

$$
\sup _{t \in[0, T]}\|P(t)\|<\infty .
$$

The Riccati equation formally associated with the deterministic control problem (28) has the form

$$
\left\{\begin{array}{l}
P^{\prime}(t)=-A^{*} P(t)-P(t) A^{*}-C^{*} C+P(t) A B B^{*} A^{*} P(t) \\
P(T)=G
\end{array}\right.
$$

but the concept of solution to this equation requires a rigorous definition. We start with some notations.

Definition 3.2. We denote by $C_{s, \alpha}([0, T] ; \Sigma(\mathcal{H}))$ the set of all $P \in C_{s}([0, T] ; \Sigma(\mathcal{H}))$ such that

$$
\begin{gathered}
\text { (i) } P(t) x \in D\left(\left(\lambda_{0}-A^{*}\right)^{1-\alpha}\right) \quad \forall x \in \mathcal{H}, \forall t \in[0, T) \\
\text { (ii) } V_{P}(t) \stackrel{\text { def }}{=}\left(\lambda_{0}-A^{*}\right)^{1-\alpha} P(t) \in C([0, T) ; \mathcal{L}(\mathcal{H})) \\
\text { (iii) } \lim _{t \rightarrow T^{-}}\left((T-t)^{1-\alpha} V_{P}(t) x\right)=0 \quad \forall x \in \mathcal{H}
\end{gathered}
$$

Given $P \in C_{s, \alpha}([0, T] ; \Sigma(\mathcal{H}))$, the norm $|P|_{\alpha}$ is defined as

$$
|P|_{\alpha} \stackrel{\text { def }}{=} \sup _{t \in[0, T)}\|P(t)\|+\sup _{t \in[0, T)}(T-t)^{(1-\alpha)}\left\|\left(\lambda_{0}-A^{*}\right)^{1-\alpha} P(t)\right\|
$$

It can be proved (see [3] page 205) that $C_{s, \alpha}\left([0, T] ; \Sigma(\mathcal{H})\right.$ ), endowed with the norm $|\cdot|_{\alpha}$, is a Banach space. We will use the notation $E \stackrel{\text { def }}{=}\left(\lambda_{0}-A\right)^{\alpha} D \in \mathcal{L}(\mathbb{R} ; \mathcal{H})$.

Note that if $|P|_{\alpha}<\infty$ then (since $\alpha>1 / 2$ )

$$
|P|_{L^{2}(0, T ; \mathcal{L}(\mathcal{H}))}<\infty
$$

Definition 3.3. We say that $P \in C_{s, \alpha}([0, T] ; \Sigma(\mathcal{H}))$ is a weak solution of the Riccati equation (30) if for all $x, y \in \operatorname{dom}(A)$ and all $t \in(0, T)$

$$
\left\{\begin{array}{l}
\frac{\mathrm{d}}{\mathrm{d} t}\langle P(t) x, y\rangle=-\langle P(t) x, A y\rangle-\langle P(t) A x, y\rangle-\langle C x, C y\rangle+\left\langle E^{*} V_{P}(t) x, E^{*} V_{P}(t) y\right\rangle \\
P(T)=G
\end{array}\right.
$$

We recall now the existence and uniqueness theorem for the (32):

Theorem 3.4. (i) The Riccati equation (32) has a unique weak solution in $P$ in $C_{s, \alpha}\left([0, T] ; \Sigma^{+}(\mathcal{H})\right)$

(ii) $P \in C_{s, \alpha}\left([0, T] ; \Sigma^{+}(\mathcal{H})\right)$ is a weak solution of (30) if and only if it solves the following mild equation:

$$
\begin{aligned}
P(t)=e^{(T-t) A^{*}} G e^{(T-t) A}+\int_{t}^{T} e^{(s-t) A^{*}} C^{*} C e^{(s-t) A} \mathrm{~d} s & \\
& +\int_{t}^{T} e^{(s-t) A^{*}} V_{P}^{*}(s) E E^{*} V_{P}(s) e^{(s-t) A} \mathrm{~d} s
\end{aligned}
$$

Proof. See [3] Theorem 2.1 page 207 for the proof of (i) and 3] Proposition 2.1 page 206 for (ii). 


\subsection{Dynamic Programming}

Lemma 3.5. We have that

$$
\int_{0}^{T}\left\langle\left(\left(\lambda_{0}-A\right) \psi_{\lambda_{0}}\right), P(t)\left(\left(\lambda_{0}-A\right) \psi_{\lambda_{0}}\right)\right\rangle_{\mathcal{H}} \mathrm{d} t<\infty
$$

Proof. We use the fact that $P$ satisfies the mild equation (33). We have that

$$
\begin{gathered}
\int_{0}^{T}\left\langle\left(\left(\lambda_{0}-A\right) \psi_{\lambda_{0}}\right), P(t)\left(\left(\lambda_{0}-A\right) \psi_{\lambda_{0}}\right)\right\rangle_{\mathcal{H}} \mathrm{d} t \\
=\int_{0}^{T} E^{*}\left(\lambda_{0}-A^{*}\right)^{1-\alpha} P(t)\left(\lambda_{0}-A\right)^{1-\alpha} E(1) \mathrm{d} t \\
=I_{1}+I_{2}+I_{3} \\
\stackrel{\text { def }}{=} \int_{0}^{T} E^{*}\left(\lambda_{0}-A^{*}\right)^{1-\alpha} e^{(T-t) A^{*}} G e^{(T-t) A}\left(\lambda_{0}-A\right)^{1-\alpha} E(1) \mathrm{d} t \\
+\int_{0}^{T} E^{*}\left(\lambda_{0}-A^{*}\right)^{1-\alpha}\left(\int_{t}^{T} e^{(s-t) A^{*}} C^{*} C e^{(s-t) A} \mathrm{~d} s\right)\left(\lambda_{0}-A\right)^{1-\alpha} E(1) \mathrm{d} t \\
+\int_{0}^{T} E^{*}\left(\lambda_{0}-A^{*}\right)^{1-\alpha} \int_{t}^{T} e^{(s-t) A^{*}} V_{P}^{*}(s) E E^{*} V_{P}(s) e^{(s-t) A} \mathrm{~d} s\left(\lambda_{0}-A\right)^{1-\alpha} E(1) \mathrm{d} t .
\end{gathered}
$$

For $I_{1}$ we have only to check the integrability for $t \rightarrow T$ and it follows from the fact that $\alpha>1 / 2$ and from (18): $\left\|\left(\left(\lambda_{0}-A^{*}\right)^{1-\alpha} e^{(T-t) A^{*}}\right)\right\| \leq M_{1-\alpha}(T-t)^{1-\alpha}$. For $I_{2}$ we proceed in a similar way: we can write $I_{2}$ as:

$$
I_{2}=\int_{0}^{T} \int_{t}^{T}\left|C\left(\left(\lambda_{0}-A\right)^{1-\alpha} e^{(s-t) A}\right) E(1)\right|^{2} \mathrm{~d} s \mathrm{~d} t
$$

and we can conclude as for $I_{1}$, using (18). For $I_{3}$ we can observe that:

$$
I_{3}=\int_{0}^{T} \int_{t}^{T}\left|E^{*}\left(\lambda_{0}-A^{*}\right)^{1-\alpha} e^{(s-t) A^{*}} V_{P}^{*}(s) E(1)\right|^{2} \mathrm{~d} s \mathrm{~d} t
$$

Note that from (ii) of Definition 3.2 and from the finiteness of the norm $|P|_{\alpha}$ we know that

$$
\left\|V_{P}^{*}(s)\right\| \leq \frac{C_{1}}{(T-s)^{1-\alpha}}
$$

and

$$
\left\|E^{*}\left(\lambda_{0}-A^{*}\right)^{1-\alpha} e^{(s-t) A^{*}}\right\| \leq \frac{C_{2}}{(s-t)^{1-\alpha}}
$$

The claim follows by straightforward computations.

Proposition 3.6. If $u \in M_{W}^{2}(\tau, T ; \mathbb{R})$ is a control and $x$ is the related trajectory, then

$$
\begin{aligned}
\mathbb{E}[\langle G x(T), x(T)\rangle+ & \left.\int_{\tau}^{T}|C x(t)|_{Y}^{2}+|u(t)|_{\mathbb{R}}^{2} \mathrm{~d} t\right] \\
=\langle P(\tau) x(\tau), x(\tau)\rangle & +\mathbb{E}\left[\int_{\tau}^{T}\left|u(t)+E^{*} V_{P}(t) x(t)\right|_{\mathbb{R}}^{2}\right] \\
& +\int_{\tau}^{T} \frac{1}{2}\left\langle\left(\lambda_{0}-A\right) \psi_{\lambda_{0}}, P(t)\left(\left(\lambda_{0}-A\right) \psi_{\lambda_{0}}\right)\right\rangle_{\mathcal{H}} \mathrm{d} t .
\end{aligned}
$$


Proof. We will perform the following steps: we first approximate $x$ using $x_{n}$ defined in (23) then we compute $\int_{\tau}^{T_{0}} \frac{\mathrm{d}}{\mathrm{d} t}\left\langle P(t) x_{n}(t), x_{n}(t)\right\rangle \mathrm{d} t$ using Ito's formula and eventually we will consider to the limit $n \rightarrow \infty$ and then $T_{0} \rightarrow T$. Let $\mathcal{L}(\operatorname{dom}(A) ; \mathcal{H})$ be the space of bounded operators from $\operatorname{dom}(A)$ endowed with the graph norm to $\mathcal{H}$. Note that since $P \in C([\tau, T) ; \mathcal{L}(\mathcal{H} ; \mathcal{H}))$ it is a fortiori and element of $C([\tau, T) ; \mathcal{L}(\operatorname{dom}(A) ; \mathcal{H}))$. Consider $T_{0}<T$ and the following function $(\operatorname{dom}(A)$ is endowed with the graph norm)

$$
\left\{\begin{array}{l}
\Phi:\left[\tau, T_{0}\right] \times \operatorname{dom}(A) \rightarrow \mathbb{R} \\
\Phi:(t, x) \mapsto\langle P(t) x, x\rangle_{\mathcal{H}}
\end{array}\right.
$$

Note that in the definition of $\Phi$ we use the scalar product of $\mathcal{H}$ and not of $\operatorname{dom}(A)$. $\Phi$ is twice continuously differentiable with locally bounded derivatives in $x$ on $\left[\tau, T_{0}\right] \times \operatorname{dom}(A)$. Moreover we have that $\partial_{x} \Phi(t, x)=P(t) x$ and $\partial_{x}^{2} \Phi(t, x)(y, z)=2\langle P(t) y, z\rangle_{\mathcal{H}}$. The first derivative in $t$ is also continuous and locally bounded on $\left[\tau, T_{0}\right] \times \operatorname{dom}(A)$. Invoking (32) we have

$$
\begin{gathered}
\frac{\mathrm{d}}{\mathrm{d} t}\langle P(t) x, y\rangle_{\mathcal{H}}=-\langle P(t) x, A y\rangle_{\mathcal{H}}-\langle P(t) A x, y\rangle-\langle C x, C y\rangle_{\mathcal{H}}+ \\
+\left\langle E^{*} V_{P}(t) x, E^{*} V_{P}(t) y\right\rangle_{\mathcal{H}} \cdot
\end{gathered}
$$

Such an expression can be discontinuous for $t=T$ only (this is the reason why we have considered a $T_{0}<T$ ). We have already observed that $x_{n}$ satisfy the integral equation (26) also in $\operatorname{dom}(A)$ and then we can use the Ito's rule (see [6] page 105): we have that

$$
\begin{gathered}
\left\langle P\left(T_{0}\right) x_{n}\left(T_{0}\right), x_{n}\left(T_{0}\right)\right\rangle=\left\langle P(\tau) x_{n}(\tau), x_{n}(\tau)\right\rangle-\int_{\tau}^{T_{0}}\left\langle C x_{n}(t), C x_{n}(t)\right\rangle_{Y} \mathrm{~d} t \\
-2 \int_{\tau}^{T_{0}}\left\langle P(t) x_{n}(t), A x_{n}(t)\right\rangle \mathrm{d} t+\int_{\tau}^{T_{0}}\left\langle E^{*} V_{P}(t) x_{n}(t), E^{*} V_{P}(t) x_{n}(t)\right\rangle_{\mathbb{R}} \mathrm{d} t \\
+2 \int_{\tau}^{T_{0}}\left\langle V_{P}(t) x_{n}(t), \mathcal{I}_{n}\left(\lambda_{0}-A\right)^{\alpha} D u(t)\right\rangle \mathrm{d} t+2 \int_{\tau}^{T_{0}}\left\langle P(t) x_{n}(t), A x_{n}(t)\right\rangle \mathrm{d} t \\
+2 \int_{\tau}^{T_{0}}\left\langle V_{P}(t) x_{n}(t), \mathcal{I}_{n}\left(\lambda_{0}-A\right)^{\alpha} D \mathrm{~d} W(t)\right\rangle \mathrm{d} t \\
\quad+\int_{\tau}^{T_{0}} \frac{1}{2}\left\langle\left(\left(\lambda_{0}-A\right) \mathcal{I}_{n} \psi_{\lambda_{0}}\right), P(t)\left(\left(\lambda_{0}-A\right) \mathcal{I}_{n} \psi_{\lambda_{0}}\right)\right\rangle_{\mathcal{H}} \mathrm{d} t .
\end{gathered}
$$

By simplifying the terms $\left\langle P(t) x_{n}(t), A x_{n}(t)\right\rangle$, adding and subtracting $|u(t)|_{\mathbb{R}}^{2}$ and $2 \int_{0}^{T_{0}}\left\langle u(t), E^{*} V_{P}(t) x_{n}(t)\right\rangle_{\mathbb{R}}$ inside the integral and taking the expectation we find:

$$
\begin{aligned}
& \mathbb{E}\left[\left\langleP\left(T_{0}\right) x_{n}\left(T_{0}\right),\right.\right.\left.\left.x_{n}\left(T_{0}\right)\right\rangle+\int_{\tau}^{T_{0}}\left|C x_{n}(t)\right|_{Y}^{2}+|u(t)|_{\mathbb{R}}^{2} \mathrm{~d} t\right] \\
&=\left\langle P(\tau) x_{n}(\tau), x_{n}(\tau)\right\rangle+\mathbb{E}\left[\int_{\tau}^{T_{0}}\left|u(t)+E^{*} V_{P}(t) x_{n}(t)\right|_{\mathbb{R}}^{2}\right] \\
&+2 \mathbb{E}\left[\int_{\tau}^{T_{0}}\left\langle V_{P}(t) x_{n}(t),\left(\mathcal{I}_{n}-I\right)\left(\lambda_{0}-A\right)^{\alpha} D u(t)\right\rangle \mathrm{d} t\right] \\
& \quad+\int_{\tau}^{T_{0}} \frac{1}{2}\left\langle\left(\left(\lambda_{0}-A\right) \mathcal{I}_{n} \psi_{\lambda_{0}}\right), P(t)\left(\left(\lambda_{0}-A\right) \mathcal{I}_{n} \psi_{\lambda_{0}}\right)\right\rangle_{\mathcal{H}} \mathrm{d} t
\end{aligned}
$$


We want now to pass with $n \rightarrow \infty$. Since by (24) we have $x_{n} \frac{C\left(\left[\tau, T_{0}\right] ; L^{2}(\Omega, \mathcal{H})\right)}{n \rightarrow \infty} x$, it is clear that

$\lim _{n \rightarrow \infty} \mathbb{E}\left(\left\langle P\left(T_{0}\right) x_{n}\left(T_{0}\right), x_{n}\left(T_{0}\right)\right\rangle+\int_{\tau}^{T_{0}}\left|C x_{n}(t)\right|_{Y}^{2} d t\right)=\mathbb{E}\left(\left\langle P\left(T_{0}\right) x\left(T_{0}\right), x\left(T_{0}\right)\right\rangle+\int_{\tau}^{T_{0}}|C x(t)|_{Y}^{2} d t\right)$

and

$$
\lim _{n \rightarrow \infty}\left\langle P(\tau) x_{n}(\tau), x_{n}(\tau)\right\rangle=\langle P(\tau) x(\tau), x(\tau)\rangle .
$$

Since $V_{P} \in C\left(\left[\tau, T_{0}\right] ; \mathcal{L}(\mathcal{H})\right)$ and $\left(\lambda_{0}-A\right)^{\alpha} D=E$ is bounded, the Dominated convergence yields

$$
\begin{gathered}
\lim _{n \rightarrow \infty} \mathbb{E}\left(\int_{\tau}^{T_{0}}\left|u(t)+E^{\star} V_{P}(t) x_{n}(t)\right|_{\mathbb{R}}^{2} d t+2 \int_{\tau}^{T_{0}}\left\langle V_{P}(t) x_{n}(t),\left(\mathcal{I}_{n}-I\right)\left(\lambda_{0}-A\right)^{\alpha} D u(t)\right\rangle d t\right) \\
=\mathbb{E} \int_{\tau}^{T_{0}}\left|u(t)+E^{\star} V_{P}(t) x(t)\right|_{\mathbb{R}}^{2} d t .
\end{gathered}
$$

Finally, using the arguments similar to those in the proof of Lemma 3.5 we obtain

$\lim _{n \rightarrow \infty} \mathbb{E} \int_{\tau}^{T_{0}} \frac{1}{2}\left\langle\left(\lambda_{0}-A\right) \mathcal{I}_{n} \psi_{\lambda_{0}}, P(t)\left(\lambda_{0}-A\right) \mathcal{I}_{n} \psi_{\lambda_{0}}\right\rangle_{\mathcal{H}} d t=\mathbb{E} \int_{\tau}^{T_{0}} \frac{1}{2}\left\langle\left(\lambda_{0}-A\right) \psi_{\lambda_{0}}, P(t)\left(\lambda_{0}-A\right) \psi_{\lambda_{0}}\right\rangle_{\mathcal{H}} d t$ and therefore, putting together the above results we obtain

$$
\begin{aligned}
\mathbb{E}\left[\left\langle P\left(T_{0}\right) x\left(T_{0}\right), x\left(T_{0}\right)\right\rangle+\int_{\tau}^{T_{0}}|C x(t)|_{Y}^{2}+|u(t)|_{\mathbb{R}}^{2} \mathrm{~d} t\right] \\
=\langle P(\tau) x(\tau), x(\tau)\rangle+\mathbb{E}\left[\int_{\tau}^{T_{0}}\left|u(t)+E^{*} V_{P}(t) x(t)\right|_{\mathbb{R}}^{2}\right] \\
+\int_{\tau}^{T_{0}} \frac{1}{2}\left\langle\left(\left(\lambda_{0}-A\right) \psi_{\lambda_{0}}\right), P(t)\left(\left(\lambda_{0}-A\right) \psi_{\lambda_{0}}\right)\right\rangle_{\mathcal{H}} \mathrm{d} t
\end{aligned}
$$

Now we pass to the limit in $T_{0} \uparrow T$ in (39). To show the convergence of the left hand side of (39) it is enough to invoke monotone convergence and to show that

$$
\lim _{T_{0} \rightarrow T} \mathbb{E}\left\langle P\left(T_{0}\right) x\left(T_{0}\right), x\left(T_{0}\right)\right\rangle=\mathbb{E}\langle P(T) x(T), x(T)\rangle .
$$

To this end note that

$$
\begin{aligned}
\langle P(T) x(T), x(T)\rangle-\left\langle P\left(T_{0}\right) x\left(T_{0}\right), x\left(T_{0}\right)\right\rangle & \\
\quad & \left\langle\left(P(T)-P\left(T_{0}\right)\right) x(T), x(T)\right\rangle \\
& +\left\langle P\left(T_{0}\right)\left(x(T)-x\left(T_{0}\right)\right), x(T)\right\rangle+\left\langle P\left(T_{0}\right) x\left(T_{0}\right), x(T)-x\left(T_{0}\right)\right\rangle .
\end{aligned}
$$

Then the strong continuity of $P$ at $T$ yields

$$
\lim _{T_{0} \rightarrow T}\left\langle\left(P(T)-P\left(T_{0}\right)\right) x(T), x(T)\right\rangle=0
$$

hence by (29) and the fact that $x \in C\left([\tau, T] ; L^{2}(\Omega ; \mathcal{H})\right)$ and the Dominated Convergence we obtain

$$
\lim _{T_{0} \rightarrow T} \mathbb{E}\left\langle\left(P(T)-P\left(T_{0}\right)\right) x(T), x(T)\right\rangle=0
$$


Again, since $x \in C\left([\tau, T] ; L^{2}(\Omega ; \mathcal{H})\right)$, we find that

$$
\left|\mathbb{E}\left\langle P\left(T_{0}\right)\left(x(T)-x\left(T_{0}\right)\right), x(T)\right\rangle\right| \leq \sup _{t \leq T}\|P(t)\|\left(\sup _{t \leq T} \mathbb{E}|x(t)|^{2}\right)^{1 / 2}\left(\mathbb{E}\left|x(T)-x\left(T_{0}\right)\right|^{2}\right)^{1 / 2}
$$

and therefore

$$
\lim _{T_{0} \rightarrow T} \mathbb{E}\left\langle P\left(T_{0}\right)\left(x(T)-x\left(T_{0}\right)\right), x(T)\right\rangle=0 .
$$

By the same arguments we obtain

$$
\lim _{T_{0} \rightarrow T} \mathbb{E}\left\langle P\left(T_{0}\right) x\left(T_{0}\right), x(T)-x\left(T_{0}\right)\right\rangle=0
$$

and therefore we obtain the convergence of the left hand side of (39). To prove convergence of the second term in the right side of (39) it is enough to show that $V_{P} x \in M_{W}^{2}(\tau, T ; \mathcal{H})$. Indeed, invoking (35) we have

$$
\begin{aligned}
\mathbb{E} \int_{0}^{T}\left|V_{P}(s) x(s)\right|^{2} \mathrm{~d} s & \leq C_{3} \int_{0}^{T}\left\|V_{P}(s)\right\|^{2} \mathbb{E}|x(s)|^{2} \mathrm{~d} s \\
& \leq C_{4}|x|_{C\left([\tau, T] ; L^{2}(\Omega ; \mathcal{H})\right)}^{2} \int_{0}^{T}\left\|V_{P}(s)\right\|^{2} \mathrm{~d} s<\infty .
\end{aligned}
$$

The convergence for the third term of the right side of (39) for $T_{0} \rightarrow T$ follows from Lemma 3.5 .

Theorem 3.7. Let $\tau \in[0, T]$ and $x_{0}$ be in $\mathcal{H}$. Then there exists a unique optimal pair $\left(u^{*}, x^{*}\right)$ at $\left(\tau, x_{0}\right)$. The optimal control $u^{*}$ is given by the feedback formula

$$
u^{*}(t)=-E^{*} V_{P}(t) x^{*}(t)
$$

and the value function of the problem is

$$
V\left(\tau, x_{0}\right)=\left\langle P(\tau) x_{0}, x_{0}\right\rangle+\int_{\tau}^{T} \frac{1}{2}\left\langle\left(\left(\lambda_{0}-A\right) \psi_{\lambda_{0}}\right), P(s)\left(\left(\lambda_{0}-A\right) \psi_{\lambda_{0}}\right)\right\rangle_{\mathcal{H}} \mathrm{d} s
$$

Proof. We begin proving that the equation

$$
x^{*}(t)=e^{(t-\tau) A} x_{0}-\int_{\tau}^{t}\left(\lambda_{0}-A\right)^{1-\alpha} e^{(t-s) A} E E^{*} V_{P}(s) x^{*}(s) \mathrm{d} s+\int_{\tau}^{t} e^{(t-s) A} B \mathrm{~d} W(s)
$$

has a unique solution and it is in $C\left([\tau, T] ; L^{2}(\Omega ; \mathcal{H})\right)$. Consider the mapping

$$
\left\{\begin{aligned}
\phi \mapsto \Psi(\phi) & \\
\Psi(\phi)= & e^{(t-\tau) A} x_{0}-\int_{\tau}^{t}\left(\lambda_{0}-A\right)^{1-\alpha} e^{(t-s) A} E E^{*} V_{P}(s) \phi(s) \mathrm{d} s \\
& +\int_{\tau}^{t} e^{(t-s) A} B \mathrm{~d} W(s) .
\end{aligned}\right.
$$


We want to prove that $\Psi(\phi)$ defines a contraction on $C\left([\tau, t] ; L^{2}(\Omega ; \mathcal{H})\right)$ if we choose $t$ small enough. Consider $\psi$ and $\phi$ in $C\left([\tau, T] ; L^{2}(\Omega ; \mathcal{H})\right)$ :

$$
\begin{aligned}
& \mathbb{E}\left[|(\Psi(\psi)-\Psi(\phi))(t)|^{2}\right] \\
& =\mathbb{E}\left[\left|\int_{\tau}^{t}\left(\left(\lambda_{0}-A\right)^{1-\alpha} e^{(t-s) A}\right) E E^{*} V_{P}(s)(\psi-\phi)(s) \mathrm{d} s\right|^{2}\right] \\
& \leq C_{1} \mathbb{E}\left[\left|V_{P}\right|_{L^{2}(0, T ; \mathcal{L}(\mathcal{H}))}^{2} \int_{\tau}^{t} \frac{1}{\left.(t-s)^{2(1-\alpha)}|(\psi-\phi)(s)|^{2} \mathrm{~d} s\right]}\right. \\
& \leq C_{2}|(\psi-\phi)(s)|_{C\left([\tau, t] ; L^{2}(\Omega ; \mathcal{H})\right)}^{2} \int_{\tau}^{t} \frac{1}{(t-s)^{2(1-\alpha)}} \mathrm{d} s
\end{aligned}
$$

where the constants $C_{1}$ and $C_{2}$ do not depend on $t$. So if $t$ is small enough $\Psi$ is a contraction on $C\left([\tau, t] ; L^{2}(\Omega ; \mathcal{H})\right)$. Similar estimates (together with the fact that $W_{A} \in C\left(\tau, T ; L^{2}(\Omega ; \mathcal{H})\right.$ ) prove that the image of $\Psi$ is in $C\left([\tau, t] ; L^{2}(\Omega ; \mathcal{H})\right)$. Proceeding by iterations (we can choose an uniform step) we have the existence and uniqueness of the solution of the (44) on $C\left([\tau, T] ; L^{2}(\Omega ; \mathcal{H})\right)$.

We will prove now that $u^{\star}$ defined by (43) is the optimal control. Its admissibility (that is $\left.u^{*} \in M_{W}^{2}(\tau, T ; \mathbb{R})\right)$ can be proved using the same argument we used in (42).

Now we observe that Proposition 3.6 implies, for every $u \in M_{W}^{2}(\tau, T ; \mathbb{R})$,

$$
J\left(\tau, x_{0}, u\right) \geq\left\langle P(\tau) x_{0}, x_{0}\right\rangle+\int_{\tau}^{T} \frac{1}{2}\left\langle\left(\left(\lambda_{0}-A\right) \psi_{\lambda_{0}}\right), P(t)\left(\left(\lambda_{0}-A\right) \psi_{\lambda_{0}}\right)\right\rangle_{\mathcal{H}} \mathrm{d} t
$$

and the couple $\left(u^{*}, x^{*}\right)$ satisfies

$$
J\left(\tau, x_{0}, u^{*}\right)=\left\langle P(\tau) x_{0}, x_{0}\right\rangle+\int_{\tau}^{T} \frac{1}{2}\left\langle\left(\left(\lambda_{0}-A\right) \psi_{\lambda_{0}}\right), P(t)\left(\left(\lambda_{0}-A\right) \psi_{\lambda_{0}}\right)\right\rangle_{\mathcal{H}} \mathrm{d} t
$$

so it is optimal. If $(\hat{u}, \hat{x})$ is another optimal couple then by (46) and (47) we have

$$
J\left(\tau, x_{0}, \hat{u}\right)=\left\langle P(\tau) x_{0}, x_{0}\right\rangle+\int_{\tau}^{T} \frac{1}{2}\left\langle\left(\left(\lambda_{0}-A\right) \psi_{\lambda_{0}}\right), P(t)\left(\left(\lambda_{0}-A\right) \psi_{\lambda_{0}}\right)\right\rangle_{\mathcal{H}} \mathrm{d} t
$$

and then (39) yields

$$
\left|\hat{u}(t)+E^{*} V_{P}(t) \hat{x}(t)\right|=0 \quad d t \otimes \mathbb{P}-\text { a.e. }
$$

and then $\hat{x}$ satisfies (44) but the solution to (44) is unique by Theorem 2.6] solution and finally we can choose continuous versions of $\hat{x}$ and $\hat{u}$ such that $x^{*}=\hat{x}$ and $u^{*}=\hat{u}$.

\section{References}

[1] N. U. Ahmed. Stochastic control on Hilbert space for linear evolution equations with random operator-valued coefficients. SIAM J. Control Optim., 19(3):401-430, 1981.

[2] E. Alòs and S. Bonaccorsi. Stochastic partial differential equations with Dirichlet whitenoise boundary conditions. Ann. Inst. H. Poincaré Probab. Statist., 38(2):125-154, 2002.

[3] A. Bensoussan, G. Da Prato, M.C. Delfour, and S.K. Mitter. Representation and control of infinite-dimensional systems. Vol. II. Systems \& Control: Foundations \& Applications. Birkhäuser Boston Inc., Boston, MA, 1993. 
[4] S. Bonaccorsi and G. Guatteri. Stochastic partial differential equations in bounded domains with Dirichlet boundary conditions. Stoch. Stoch. Rep., 74(1-2):349-370, 2002.

[5] G. Da Prato. Direct solution of a Riccati equation arising in stochastic control theory. Appl. Math. Optim., 11(3):191-208, 1984.

[6] G. Da Prato and J. Zabczyk. Stochastic equations in infinite dimensions. Encyclopedia of Mathematics and Its Applications. 44. Cambridge etc.: Cambridge University Press. xviii, 454 p., 1992.

[7] G. Da Prato and J. Zabczyk. Evolution equations with white-noise boundary conditions. Stoch. Stoch. Rep., 42(3-4):167-182, 1993.

[8] A. Debussche, M. Fuhrman, and G. Tessitore. Optimal control of a stochastic heat equation with boundary-noise and boundary-control. ESAIM Control Optim. Calc. Var., 13(1):178-205 (electronic), 2007.

[9] T. E. Duncan, B. Maslowski, and B. Pasik-Duncan. Adaptive boundary control of stochastic linear distributed parameter systems described by analytic semigroups. Appl. Math. Optim., (33):107-138, 1996.

[10] F. Flandoli. Direct solution of a Riccati equation arising in a stochastic control problem with control and observation on the boundary. Appl. Math. Optim., 14(2):107-129, 1986.

[11] N.V. Krylov. Weighted Sobolev spaces and Laplace's equation and the heat equations in a half space. Comm. Partial Differential Equations, 24(9-10):1611-1653, 1999.

[12] N.V. Krylov. The heat equation in $L_{q}\left((0, T), L_{p}\right)$-spaces with weights. SIAM J. Math. Anal., 32(5):1117-1141, 2001.

[13] I. Lasiecka. Unified theory for abstract parabolic boundary problems: a semigroup approach. Appl. Math. Optim., 6(1):287-333, 1980.

[14] I. Lasiecka and R. Triggiani. Control theory for partial differential equations: continuous and approximation theories. I. Abstract parabolic systems, volume 74 of Encyclopedia of Mathematics and its Applications. Cambridge University Press, Cambridge, 2000.

[15] J.L. Lions and E. Magenes. Non-homogeneous boundary value problems and applications. Vol. I, volume 181 of Die Grundlehren der mathematischen Wissenschaften. SpringerVerlag, New York, 1972.

[16] C. Martínez Carracedo and M. Sanz Alix. The theory of fractional powers of operators, volume 187 of North-Holland Mathematics Studies. North-Holland Publishing Co., Amsterdam, 2001.

[17] B. Maslowski. Stability of semilinear equations with boundary and pointwise noise. Ann. Scuola Norm. Sup. Pisa Cl. Sci. (4), 22(1):55-93, 1995.

[18] A. Pazy. Semigroups of linear operators and applications to partial differential equations, volume 44 of Applied Mathematical Sciences. Springer-Verlag, New York, 1983. 